[Bull. Agr. Chem. Soc. Japan, Vol. 20, No. 3, p. 106 109, 1956]

\title{
Einwirkung von Äthylenoxyden auf Lysozym
}

\author{
Von Kazuyuki Maekawa und Masahisa Kushibe \\ Institul fïr biologische Chemie der Landwirtschaftlichen Fakuliät, Universität Ehime
}

Eingegangen am 9, Januar 1956

\begin{abstract}
One mol. of lysozyme combined with 12 mols. of ethylene oxide at $4-5^{\circ} \mathrm{C}, \mathrm{pH} 8.7$, when magnesium ion existed.

Lysozyme, after having reacted with ethylene oxide so thoroughly that amino groups were no more detectable, still did not alter in the lytic activity and UV-absorption. Only in the ionophoresis a different result appeared. This $\mathrm{N}$-hydroxyethyl derivative of lysozyme behaved also almost equally with the native lysozyme in the oxidation by periodate.
\end{abstract}

Schroeder berichtete, dass die Aktivität vom Lysozyme fast zurückbleibt, als man dessen Aminogruppe mit 2,4-Dinitrofluorobenzol blockiert1). D. De Fontaine et al. liessen Phenylisothiocyanat auf Lysozym reagieren, um dessen Amino-Ende zu bestimmen, aber sie beschrieben nichts weiter in Bezug auf die Veränderung seiner Aktivität ${ }^{2}$. Neuerdings behandelten Dickman et al. das Lysozym mit Xanthydrol und bekamen Xanthyllysozyme, welches im geraden Verhältnisse zu der Mange zugesetztes Xanthydrols seine Aktivität verliert, und weiter durch Hydrolyse desselben Monoxanthyltryptophan ${ }^{3}$.

Hinsichtlich der Reaktion des Äthylenoxyds auf Lysozym gibt es jedoch neuerdings keinen Versuch. Wir beschäftigten uns also mit der Reaktion von Lysozyme mit Äthylenoxyde nach der Weise, wie in vorläufiger Mitteilung vorgestellt ist, um seine Aminogruppe zu blockieren.

Das so zur Genüge verbundene Lysozyme, wie keine freien Aminogruppen mehr erkennbar sind, hat auch gänzliche Aktivität und zeigt im Vergleich mit nativem Lysozyme ganz ähnliches Absorptions-spektrum. Es verhält sich aber verschiedentlich bei der

1) W. A. Schroeder; J. Am, Chem. Soc., 74, 5118 (1952).

2) D. De Fontaine und S. W. Fox; ebenda, 76, 3701 (1954).

3) S. R. Dickman, R.B. Kropf und C. M. Proctor; J. Biol. Chem., 210, 491 (1954).
Papierionophorese; das behandelte Lysozym wandert nämlich bei $\mathrm{pH} 4.0$ nicht, während Lysozyme sich nach Kathode fortbewegt. (Fig. 1)

In dem Versuche von Perjodat-Oxydation des mit Äthylenoxyde blockierten Lysozyme kamen wir zum gleichen Resultate mit den schon von uns berichteten Ergebnissen über das Lysozym4).

Aus diesen Tatsachen lässt sich folgern, dass sowohl die am Ende stehenden Amino-

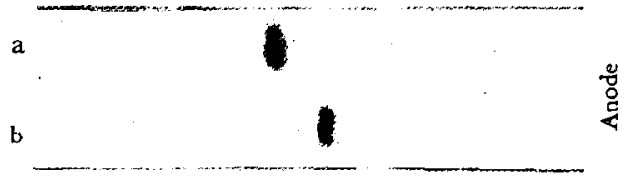

FIG. 1. Elektrophoregramm vom ÄO-Lysozyme.

a) Kontrol (natives Lysozym)

b) $\ddot{A} O \cdot L y s o z y m$

Michaelis'cher N/IO Tartrate-Puffer (pH 4.0), 500 Volt, 4.8-9.5 mA/4 cm, $3 \mathrm{Stdn}$. $\mathrm{HgCl}_{2}$-BPB-Färbung.

gruppe, einschliesslich $\varepsilon$-Aminogruppe, als auch die Oxyaminosäure mit lytischer Aktivität keinesfalls zu tun haben.

\section{BESCHREIBUNG DER VERSUCHE}

(a) Reaktion des Äthylenoxyds auf Lysozym. Mehrfach umkristallisiertes Lysozym wurde in verdünnter Essigsäure von $\mathrm{pH} 4.5$ derart aufgelöst, dass die Konz. der Enzymlösung bei 5-7 mg/ml blieb

4) K. Maekawa und M. Kushibe; Bull. Agric. Chem. Soc. Japan, 19, 28 (1955). 
Einwirkung von Athylenoxyden auf Lysozym

TABELLE I

REAKTION DES LYSOZYMS MIT ÄTHYLENOXYDE

(Ansatz der Versuche)

$\begin{array}{ccc}\text { Versuchs } & \text { Lysozym } & \text { Zugesetzte } \\ \text { Nr. } & \text { Lösung* } & \text { AO Lösung** } \\ 1 & 10 & 5 \\ 2 & 10 & 5 \\ 3 & 10 & - \\ 4 & - & 5 \\ 5 & - & 5\end{array}$

\begin{tabular}{c} 
Zugesetztes \\
Mg*" \\
1 \\
- \\
1 \\
\hline
\end{tabular}

Puffer Lösung ( $\mathrm{pH}$ 8.7)

Verbundenes

Versuchs Reaktions-

AO $\mathrm{Mol}$

Nr. dauer
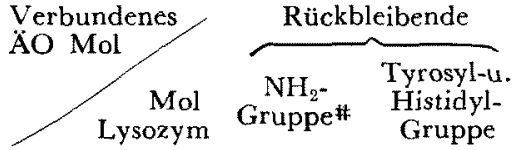

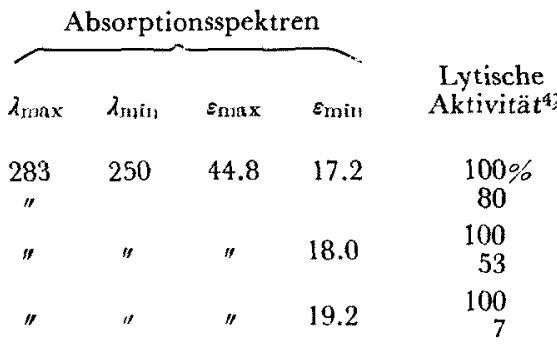

* Die Enzymlösung enthält $6.42 \mathrm{mg}$ Protein/ml.

* Die Lösung enthält $60 \mathrm{mg} \ddot{\mathrm{AO}} / \mathrm{ml}$.

\# Es wurde mittels Ninhydrins bestimmt.

\# \#s wurde danks Folinsches Phenol-reagens bestimmt.

(nach Biuret), dann mit $\mathrm{NaOH}$ auf $\mathrm{pH} 8.7$ eingestellt.

$\mathrm{Zu} 10 \mathrm{ml}$ dieser Lysozymlösung wurde $5 \mathrm{ml}$ des beinahe $40-60 \mathrm{mg} / \mathrm{ml}$ Äthylenoxyd ( $\ddot{\mathrm{AO}}$ ) haltenden Wassers zugesetzt, dann mit $1 \mathrm{ml}$ von $\mathrm{MgCl}_{2}$ Lösung versetzt, die $100 \mathrm{mg} / \mathrm{ml} \mathrm{Mg*}$ enthielt und vorher auf pH 8.7 eingestellt worden war (Tabelle I). Das Reaktionsgemisch wurde bei 4-5 $\mathrm{C}$ unter gelegentlicher Pipettierung gelassen. Die ausgezogene Lösung in Gebrauch nehmend, bestimmte man die Menge von verbundenem $\ddot{A O}$, die lytische Aktivität und die freie $\mathrm{NH}_{2}$-Gruppe.

Wie man aus der Tabelle I erkannt, verbanden $12 \mathrm{Mol}$ von ÄO sich bei $\mathrm{pH} 8.7 \mathrm{mit} 1 \mathrm{Mol}$ vom Lysozyme und wurde die freie $\mathrm{NH}_{2}$-Gruppe des Lysozyms völlig einwickelt, aber die anfängliche Aktivität blieb unverändert, während ein ziemlich grosser Abstand sich hinsichtlich der Veränderung von der Aktivität im Verlaufe der Zeit zwischen dem mit $\ddot{A O}$ behandelten Lysozyme und nativem Lysozyme befand (Figur 2). Bewahrt man es ohne Zusatz von $\mathrm{Mg}-$ Verbindung während verhältniśsmässig läng'erè Dauer aur, so reagiert $\ddot{\mathrm{AO}}$ ein wenig auf $\mathrm{NH}_{2}$-Gruppe und freie $\mathrm{NH}_{2}$-Gruppe vermindert sich einigermassen, während seine Aktivität bei weitem abnimmt. Also ist $\mathrm{Mg}$-Salz sowohl ein Katalysator bei der Reaktion des $\tilde{\mathrm{A}}$, als auch spielt es für Beständigkeit vom Lysozyme eine wichtige Rolle.

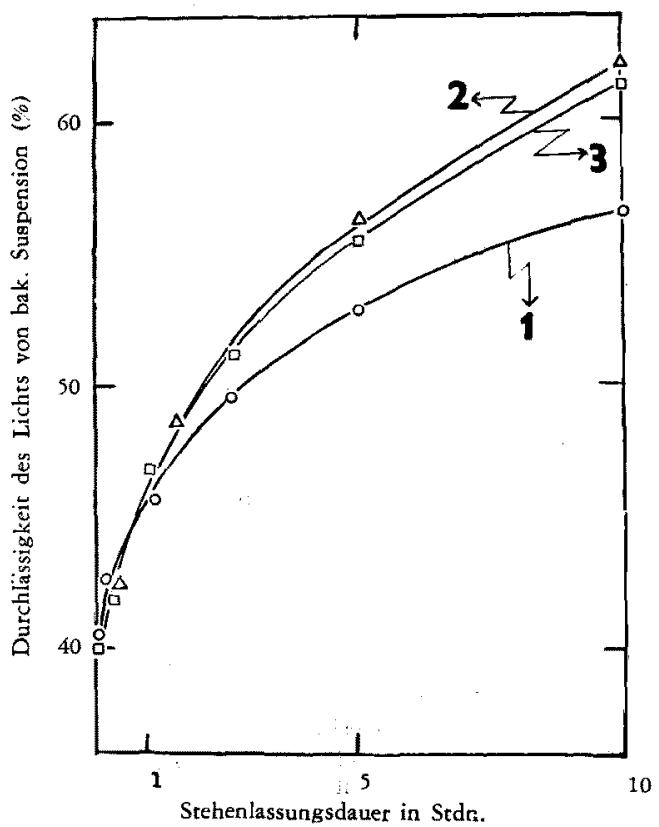

FIG. 2. Veränderung von der Aktivität im Verlaufe der Zeit bei dem mit $\mathrm{AO}$ behandelten Lysozyme.

1. $\ddot{A} \mathrm{O}-\mathrm{Lysozym}$ (mit Mg*) $6.975 r$ Enzym

2. Natives Lysozym (mit $\mathrm{Mg}^{*}$ ) $6.965 \%$ "

3. Lysozym und AO (ohne $\mathrm{Mg}^{*}$ ) 100r " 
Reaktionsgemisch (Lysozym, ÄO und Mg-Salz)

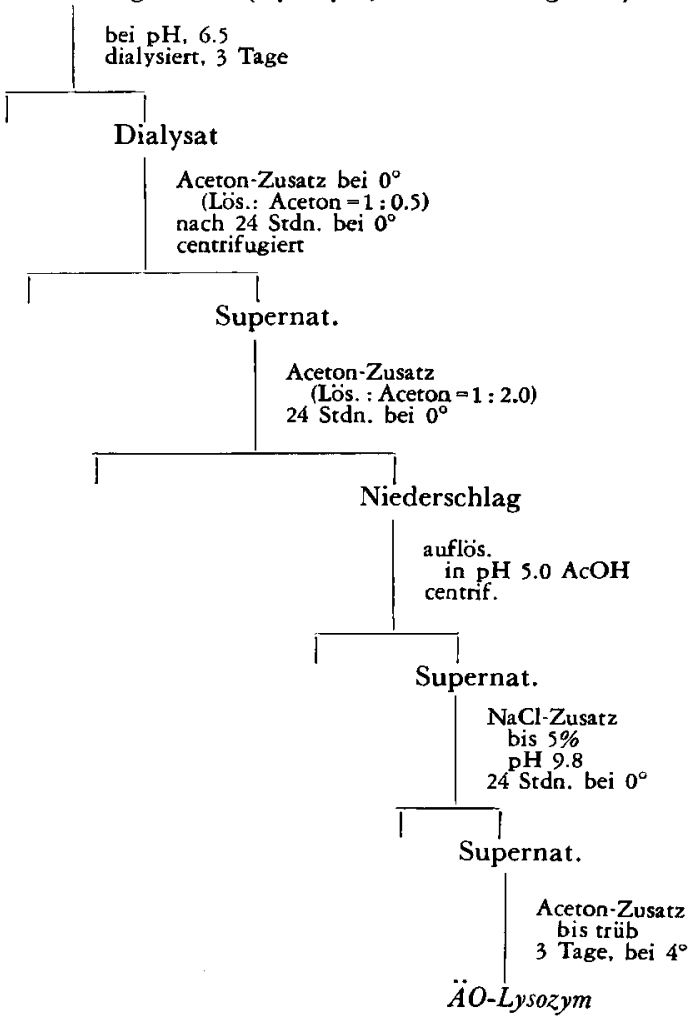

FIG. 3. ÄO-Lysozym (lytische Aktivität 100\%, freie $\mathrm{NH}_{2}$-Gruppe gedeckt).
Isolierung des mit $\ddot{A} O$ behandellen Lysozyms.

Die mit $\mathrm{AO}$ nach oben erwähnter Weise behandelte Lysozymlosung wurde fraktioniert, wie folgendes Schema zeigt, um modifiziertes Lysozym zu isolieren. Schliesslich konnten wir mit geringer Ausbeute das Lysozym-Derivate erhalten.

(b) Oxydation des mit $\ddot{A} O$ behandelten Lysozyms durch $\mathrm{NaJO}_{4}$. Das modifizierte Lysozym, in welchem nicht mehr die freie $\mathrm{NH}_{2}$-Gruppe vorhanden war und welches doch völlige Aktivität besass, wurde der Perjodat-Oxydation unterworfen.

Aus dem genug reagierten Gemische (2 Wochen lang stehen gelassen) wurde das überschüssige $\mathrm{AOO}$ durch Lüftung beseitigt, dann wurde die Lösung mit verd. $\mathrm{AcOH}$ wieder auf $\mathrm{pH} 5.3$ eingestellt. $\mathrm{Zu} 10 \mathrm{ml}$ von dieser Enzymlösung, nach Abkühlen bis $0 \mathrm{C}$, setzte man die berechnete Menge von abgekühlter $0.05 \mathrm{~N}$ $\mathrm{NaJO}_{4}$ Lösung (0.1 N Acetat-Puffer von $\mathrm{pH} 5.3$ ) zu. Das Reaktionsgemisch wurde bei 4-5 $\mathrm{C}$ stehen gelassen. Nachdem Perjodat, nötigenfalls vermittels Hinzufügens von wenigem Traubenzucker, reduziert worden war, wurde die Lösung mit Kochsalz, dessen Konzentration bis $5 \%$, dann mit verd. Natronlauge versetzt, um Enzyme niederzuschlagen. Der dabei entstandene Niederschlag wurde centrifugiert und mit der $5 \%$ iges Kochsalz enthaltenden, abgekühlten verd. Natronlauge von pH 9.5 wiederholt gewaschen, bis es von Halogen frei wurde, und zuletzt wieder in verd. Essigsäure von $\mathrm{pH} 5.4$ aufgelöst. Mit der dabei erhaltenen Lösung mass man die enzymatiche Aktivität und das Absorptionsspektrum.

Die Tabelle II gibt die dabei erhaltenen Ergebnisse an. Aus dieser Tabelle ist zu ersehen, dass die mit ÄO behandelte Probe sich bei der Oxydation durch $\mathrm{NaJO}_{4}$ ganz ähnlich mit dem Resultate über natives Lysozym benimmt. Das Verschwinden der Aktivität und die Verschiebung von UV-Absorptionsspektren standen auch jede für sich in näherer Beziehung; es befindet sich nämlich wesentlicher Unterschied nicht.

TABELLE II

OXYDATION DES MIT ÄO BEHANDELTEN LySOZYMS MIT PERJODATE

\begin{tabular}{|c|c|c|c|c|c|c|c|}
\hline \multirow[b]{2}{*}{$\begin{array}{c}\text { Versuchs } \\
\text { Nr. }\end{array}$} & \multirow[b]{2}{*}{$\begin{array}{l}\text { Lysozym } \\
\text { od. sein } \\
\text { Derivat. }\end{array}$} & \multicolumn{3}{|c|}{ Nach 8 stdg. Oxydation } & \multicolumn{3}{|c|}{ Nach 22 stdg. Oxydation } \\
\hline & & $\begin{array}{l}\text { Verbr. } \\
\mathrm{NaJO}_{4} \\
\mathrm{Mol}\end{array}$ & $\begin{array}{l}\text { Lytische } \\
\text { Aktivität }\end{array}$ & $\begin{array}{c}\text { Extinktion beim } \\
\text { Gipfel von } \\
280.5 \mathrm{~m} \mu\left(\varepsilon \times 10^{-4}\right)\end{array}$ & $\begin{array}{l}\text { erbr. } \\
\mathrm{TaJO}_{4} \\
\mathrm{Aol}\end{array}$ & $\begin{array}{l}\text { Lytische } \\
\text { Aktivität }\end{array}$ & $\begin{array}{c}\text { Extinktion beim } \\
\text { Gipfel von } \\
280.5 \mathrm{~m} \mu\left(\varepsilon \times 10^{-1}\right)\end{array}$ \\
\hline 1 & ÄO-Lysozym & $3.1 *$ & $43 \%$ & 2.5 & 7.8 & $3.4 \%$ & 0.85 \\
\hline 3 & $\begin{array}{l}\text { natives } \\
\text { Lysozym }\end{array}$ & 3.3 & $43 \%$ & 2.5 & 7.8 & 3.8 & 0.85 \\
\hline
\end{tabular}


Man kann also bei der Perjodat-Oxydation keinen Einfluss wegen der Verbindung von $\dddot{\mathrm{A} O}$ erkennen.

\section{DISKUSSION}

Da die Aminogruppe bei pH 8.7 zum Teile als $\mathrm{NH}_{2}$ vorhanden ist, musste $\ddot{\mathrm{AO}}$ auf diesem Teile reagieren. Es ist auch offenbar aus der Farbreaktion mittels Phenol-reagens, dass $\ddot{\mathrm{AO}}$ zur Oxygruppe von Tyrosine nichts bedeutet. Es lässt sich aus der vorhergehenden Mitteilung folgern, dass $\ddot{\mathrm{AO}}$ mit aliphatischer Oxygruppe nichts zu tun hat.

Sowohl unser Versuch, dass freie $\mathrm{NH}_{2}$ Gruppe nicht mehr erkennbar ist und doch volle Aktivität aufbewahrt worden ist, als auch das Ergebnis von Schroederil liessen uns zu dem Meinung kommen, dass freie $\mathrm{NH}_{2}$-Gruppe von Lysozyme mit seiner Aktivität in keiner Beziehung stehes).

Wenn $-\mathrm{CH}_{2} \mathrm{CH}_{2} \mathrm{OH}$ anstatt $\mathrm{H}$ von $\mathrm{NH}_{2}-$

5) H. Neurath und K. Balley; The Proteins, Vol. 11. S. 465 (1954).
Gruppe sich verband, so muss die Glykolspaltung von den Oxyaminosäuren schwierig sein. Jedoch wurde wirklich das mit $\ddot{A O}$ behandelte Lysozym in gleichem Benehmen mit nativem Lysozyme durch $\mathrm{NaJO}_{4}$ oxydiert und seine Aktivität verlor.

Es lässt sich also erkennen, dass nicht nur $\mathrm{NH}_{2}$-Gruppe, sondern auch die Oxygruppe von ihrem benachbarten Kohlenstoffe mit der Aktivität nichts $z$ t tun haben.

\section{ZUSAMMENF ASSUNG}

Lysozym wurde in Anwesenheit von Magnesiumsalz bei pH 8.7 mit $\ddot{A O}$ behandelt. Dadurch liess sich angeben, dass $12 \mathrm{Mol}$ von $\ddot{\mathrm{AO}}$ sich mit pro Mol Lysozyme verbanden, freie $\mathrm{NH}_{2}$-Gruppe völlig entschwand, aber die Aktivität und das Spektrum die keine merkliche Änderung zeigten. Im Falle, dass das mit $\ddot{A} O$ behandelte Lysozym durch $\mathrm{NaJO}_{4}$ oxydiert wurde, verhielt es sich mit dem nativen Lysozyme analogisch. 